Canadian Oncology

Nursing Journal

Revue canadienne

de soins infirmiers

en oncologie

Volume 30, Issue 4 • Fall 2020

elSSN: 2368-8076 


\section{Optimiser les soins palliatifs précoces aux patients atteints d'un cancer hématologique recevant une greffe de cellules souches : rôle de l'infirmière praticienne}

par Ellen A. Lewis

RÉSUMÉ

La greffe de cellules souches hématopoiétiques (GCSH) est une approche thérapeutique intensive des hémopathies malignes qui s'accompagne d'un important risque de morbidité et de mortalité. Malgré la lueur d'espoir qu'elle apporte aux patients, les traitements sont longs et pénibles, tant du point de vue physique que psychosocial. Habituellement, la greffe de cellules souches hématopoiétiques a une visée curative qui laisse peu de place à un partenariat éventuel avec les services de soins palliatifs et, lorsque ces derniers sont proposés, il est souvent trop tard pour en tirer un bénéfice notable. Il faudrait donc systématiser le recours aux soins palliatifs pour les patients atteints d'hémopathie maligne qui reçoivent une GCSH; cependant, il demeure difficile de trouver la meilleure voie pour établir un tel partenariat. Les infirmières praticiennes, qui sont de plus en plus nombreuses, possèdent les connaissances et les compétences requises pour combler les lacunes dans la prestation de soins palliatifs aux patients recevant une GCSH. L'algorithme de soins palliatifs précoces dispensés par les infirmières praticiennes aux patients recevant une GCSH (dit NEST en anglais, pour " Nurse-Practitioner-Delivered Early Palliative Care for Stem Cell Transplant ») vise justement à faire le pont entre les services palliatifs et les équipes d'hématologie et de greffe afin d'offrir aux patients les meilleurs soins possible.

Mots-clés : qualité de vie, greffe de cellules souches hématopoiétiques, soins palliatifs, soins palliatifs précoces, infirmière praticienne

\section{INTRODUCTION}

T a prestation simultanée de soins palliatifs et de soins ـoncologiques, préconisée dans les dernières recherches, présente bien des avantages : elle bonifie la qualité de vie, améliore la survie globale et réduit le coût des soins de santé (El-Jawarhi et al., 2016; Ferrell et al., 2017; May et al., 2017; Temel et al., 2010). Récemment, des auteurs ont mis en lumière l'important rôle que peuvent jouer les infirmières praticiennes (IP) pour offrir des soins palliatifs aux patients en oncologie (Dyar, Lesperance, Shannon, Sloan et Colon-Otero, 2012; Mitchell et al., 2016; Owens et al., 2012).

\section{AUTEURE}

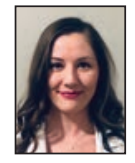

Ellen A. Lewis, inf. aut. B.Sc.inf., M.Sc.inf., IP, infirmière praticienne, hématologie, Tom Baker Cancer Centre, Foothills Medical Centre, AB

Ellen.lewis@ahs.ca
Malgré les avantages avérés, l'offre de soins palliatifs aux patients en hématologie pose un défi particulier, surtout pour les patients en greffe de cellules souches hématopoïétiques (GCSH), car la maladie évolue de façon imprévisible; il est donc difficile de savoir quand faire appel aux soins palliatifs (Levine, Baker, Wolfe, Lahmann et Ullrich, 2017). Même si l'Association médicale canadienne (AMC), le National Comprehensive Cancer Network (NCCN) et l'American Society of Clinical Oncology (ASCO) reconnaissent les avantages des soins palliatifs pour les patients en oncologie, l'intégration des soins palliatifs précoces (SPP) est rarement systématique et la disparité est encore plus apparente en hématologie (Canadian Medical Association/Association médicale canadienne, 2015; Dans et al., 2017; El-Jawahri et al., 2016; Ferrell et al., 2017).

Le présent article recense la recherche sur l'importance des SPP pour les patients en hématologie et les receveurs d'une GCSH, de même que les avantages d'avoir recours aux IP en soins palliatifs. La figure 1 propose un cheminement clinique intégrant les soins palliatifs dispensés par les IP aux soins usuels des patients recevant une GCSH.

\section{CONTEXTE}

La GCSH est souvent l'une des seules approches dont le but est de guérir l'hémopathie maligne ou de la contrôler à long terme. Toutefois, il s'agit d'un traitement intensif pouvant entraîner de la toxicité pour les organes, un échec de la greffe, un lourd fardeau de symptômes, une piètre qualité de vie et un risque élevé de mortalité (Chung, Lyckholm et Smith, 2009; El-Jawahri et al., 2015). Bien que la GCSH améliore l'état de santé des patients, le traitement s'accompagne souvent d'une chimiothérapie vigoureuse et prolongée, sans compter le risque de décès des suites d'une infection ou de la défaillance d'un organe (D'souza, Lee, Zhu et Pasquini, 2017; Levine et al., 2017). Lorsque les approches thérapeutiques échouent, il n'est pas rare que les patients subissent, dans leurs dernières semaines de vie, des traitements intensifs qui nécessitent une hospitalisation aux soins intensifs et une chimiothérapie de rattrapage (Ho et al., 2011; Hui et al., 2014). Étant donné l'évolution imprévisible de la maladie et la difficulté de savoir quand faire appel aux services de soins palliatifs spécialisés, les patients en hématologie et leur famille sont parfois pris au dépourvu lorsque la mort devient inévitable ou qu'une importante morbidité est présente (Levine et al., 2017).

Récemment, des auteurs ont proposé d'offrir des services de soins palliatifs aux receveurs d'une GCSH parallèlement 


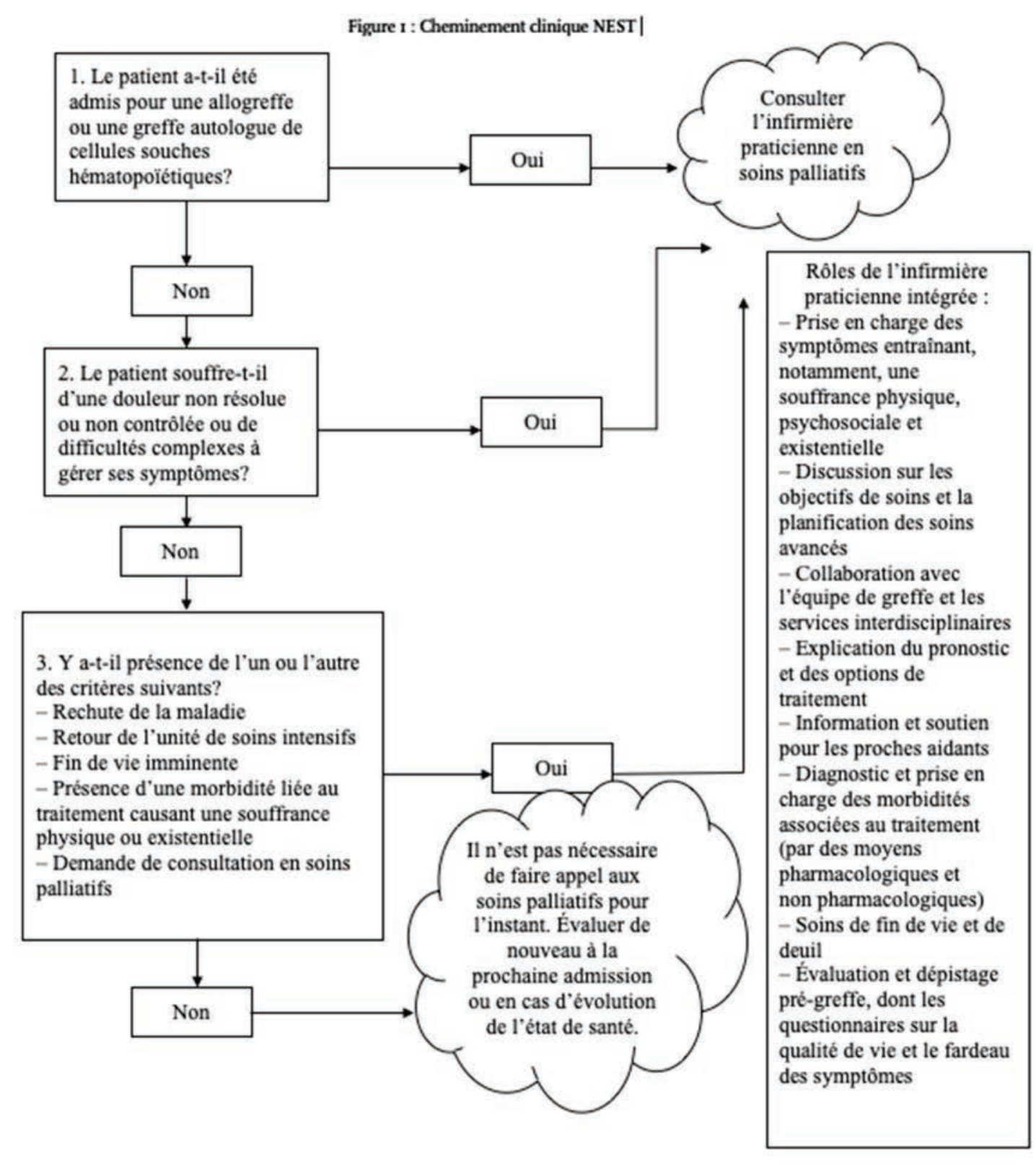

Figure 1 : Cheminement clinique NEST

aux soins curatifs (El-Jawahri et al., 2016; Harden et Schembri, 2016; Loggers et al., 2016; Roeland et Ku, 2015; Selvaggi et al., 2014). De son côté, l'ASCO a établi des lignes directrices recommandant le recours aux soins palliatifs dans les huit semaines suivant le diagnostic lorsque les symptômes physiques ou psychosociaux sont difficiles à contrôler ou que le cancer est avancé (métastases à distance, stade avancé, pronostic de 6 à 24 mois ou cancer limitant l'espérance de vie; Ferrell et al., 2017; Smith et al., 2012). De récents projets pilotes ont prouvé l'efficacité des IP pour prodiguer les soins palliatifs; elles constituent donc un choix optimal pour faciliter la prestation de SPP aux receveurs d'une GCSH (Dyar et al., 2012; Mitchell et al., 2016; Owens et al., 2012).

Offerts par une équipe de membres spécialement formés, les services de soins palliatifs spécialisés favorisent et assurent la qualité de vie des patients et de leurs proches aidants. Euvrant auprès des patients, ces équipes de soins sont en place pour soulager les symptômes physiques et existentiels, pour bien faire comprendre la maladie, le pronostic, les objectifs du traitement et tout ce qui a trait aux volontés ainsi que pour aider à la prise de décision (Ferrell et al., 2017). Malheureusement, ce type de soins est souvent associé uniquement à la fin de vie et, jusqu'à tout récemment, il n'était pas considéré comme complémentaire aux soins curatifs.

\section{REVUE DE LA LITTÉRATURE}

Les publications sur les IP, les SPP et l'oncologie ont fait l'objet d'une recherche systématique dans les bases de données Cumulative Index of Nursing and Allied Health Literature (CINAHL), MEDLINE, PubMed et Google Scholar. Les thèmes de recherche étaient les suivants : soins palliatifs, soins palliatifs précoces, soins de fin de vie et soins de soutien, en 
conjonction avec les termes cancer, tumeur maligne, néoplasie, oncologie, greffe de moelle osseuse, greffe de cellules souches, greffe hématopoiétique, hématologie et cancer avancé. Des recherches supplémentaires sur les IP ont été menées à l'aide des termes « infirmière praticienne » et « infirmière en pratique avancée », en association avec les thèmes de recherche « oncologie » et « soins palliatifs » déjà mentionnés précédemment. N'étaient conservées que les publications anglaises ultérieures à 1990. La bibliographie des articles retenus a été passée en revue pour vérifier l'inclusion de toute la littérature pertinente.

\section{Soins palliatifs précoces : état de la question}

L'offre de SPP aux patients atteints du cancer peut améliorer leur qualité de vie et leur survie globale, diminuer le recours aux interventions lourdes en fin de vie, et réduire le coût des soins de santé (Bakitas et al., 2009; Ho et al., 2011; May et al., 2017; Temel et al., 2010; Temel et al., 2011; Zimmermann et al., 2014). En 2010, Temel et collaborateurs ont réalisé un essai clinique randomisé monocentrique auprès de 151 patients souffrant d'un cancer du poumon non à petites cellules au stade métastatique, puis comparé les effets des SPP ( $n=77)$ aux soins oncologiques usuels $(n=74)$. Ils ont alors constaté une amélioration statistiquement significative de la qualité de vie des patients ayant reçu des SPP selon trois échelles de mesure distinctes : l'échelle d'évaluation fonctionnelle du cancer ou Functional Assessment of Cancer Scale $(p=.03)$, la sous-échelle du cancer du poumon ou Lung Cancer Subscale $(p=.04)$ et l'index des résultats d'essais ou Trial Outcome Index $(p=.04)$. Entre autres résultats, ils rapportent également une survie globale statistiquement plus longue, c'est-à-dire 11,6 mois contre 8,9 mois $(p=.02)$, de même que des soins de fin de vie moins intensifs (54\% [n=56] vs $33 \%$ $[n=49] ; p=.05)$ dans le groupe recevant des SPP. Dans une autre étude de Temel et collaborateurs (2011), les patients recevant des SPP avaient une perception plus juste de leur pronostic comparativement au groupe bénéficiant des soins habituels $(82,5 \%[n=57]$ contre 59,6\% [n=47]; $p=.02)$. Ces travaux de Temel et collaborateurs (2010 et 2011) sur les avantages des SPP ont par la suite inspiré de nombreuses autres études sur les bienfaits des soins palliatifs précoces (Bakitas et al., 2015; May et al., 2017; Zimmerman et al., 2014).

Zimmermann et collaborateurs (2014) ont mené un essai clinique randomisé auprès de 461 patients en oncologie atteints d'une tumeur solide. Les patients étaient répartis aléatoirement en un premier groupe qui recevait des SPP $(n=228)$ et un second groupe, les soins oncologiques usuels $(n=233)$. Ils ont constaté une différence statistiquement significative dans la qualité de vie après trois mois $(p=.05)$ et quatre mois $(p=.003)$ en faveur du groupe bénéficiant des SPP (Zimmermann et al., 2014). Un autre essai clinique randomisé, le projet ENABLE III réalisé par Bakitas et collaborateurs (2015) auprès de 207 patients atteints de cancer avancé assignés aléatoirement à un groupe recevant des soins palliatifs précoces (un à deux mois après le diagnostic; $n=104$ ) ou des soins palliatifs tardifs (trois mois ou plus après le diagnostic; $n=103$ ), a noté une augmentation statistiquement significative de la survie globale après un an chez les patients décédés à la fin de la période de collecte de données $(n=109)$ qui avaient reçu des SPP (63\% [ $n=59]$ contre $48 \%[n=50] ; p=.038)$. Une étude de cohorte prospective multicentrique menée par May et collaborateurs (2017) a mis en lumière d'autres avantages des SPP. Cette étude regroupait 863 patients atteints d'un cancer avancé recevant des soins usuels ( $n=637$ ), des soins palliatifs précoces (dans les deux jours suivants l'admission; $n=177$ ) ou des soins palliatifs tardifs (plus de deux jours après l'admission; $n=49$ ). Pour le groupe recevant des SPP, May et collaborateurs (2017) ont constaté une baisse des dépenses par hospitalisation (de 1539 \$ US) attribuables à la réduction de la durée du séjour (968 \$ US [63\%]) et de l'intensité des traitements (571 \$ US [37\%]).

La vaste majorité de la recherche sur les SPP porte sur les patients en oncologie atteints de tumeurs solides, mais on peut présumer que les soins palliatifs précoces seraient également bénéfiques pour les patients en hématologie recevant une GCSH (Loggers et al., 2016). Dans une analyse transversale multicentrique regroupant 1235 patients atteints du cancer, Hochman et collaborateurs (2018) ont comparé la nécessité de recourir aux soins palliatifs pour soulager les symptômes de patients en hématologie $(n=108)$ et de patients en oncologie atteints d'une tumeur solide ( $n=1$ 127). Ils n'ont noté aucune différence statistiquement significative entre les groupes en ce qui concerne la douleur, l'anxiété, la dyspnée, la constipation, la nausée, la dépression, l'appétit et le bienêtre; toutefois, pour les patients en hématologie, le risque était statistiquement plus élevé d'éprouver de la fatigue (rapport de cotes de 2,19; intervalle de confiance à $95 \% 1,22-3,91$; $\mathrm{p}<.01$ ) et de la somnolence (rapport de cotes de 1,81; IC $95 \%$ 1,07-3,07; $\mathrm{p}<.03$ ) comparativement aux patients en oncologie atteints d'une tumeur solide (Hochman et al., 2018).

Même si les patients ont les mêmes besoins, , le taux de renvoi vers les soins palliatifs pour les patients en hématologie est inférieur, une donnée troublante. Dans leur méta-analyse, Howell et collaborateurs (2010) ont noté qu'il était moins probable que ces patients reçoivent des soins palliatifs spécialisés comparativement aux patients en oncologie atteints d'une tumeur solide (risque relatif de 0,46; IC $95 \%, 0,42-0,50$ ). De plus, dans une étude à méthodologie mixte, LeBlanc et collaborateur (2015) ont comparé, les habitudes des hématologues $(n=23)$ et des oncologues $(n=43)$ au moment d'orienter les patients vers les services de soins palliatifs; ils ont constaté que les hématologues associaient davantage les soins palliatifs à la fin de vie $(61 \%[n=14]$ contre $16 \%,[n=6] ; p=.001)$. En effet, $30 \%(n=7)$ des hématologues n'avaient jamais dirigé de patients vers les soins palliatifs $(p=.001)$. En résumé, systématiser le recours aux SPP est impératif dans les cas d'hémopathie maligne étant donné l'intensité accablante de la GCSH, la probabilité de lourds soins en fin de vie, et le faible taux de renvoi vers les soins palliatifs. Les SPP ainsi systématisés pourraient améliorer la qualité de vie des patients.

\section{Soins palliatifs précoces et GCSH}

El-Jawahri et collaborateurs (2015) ont réalisé les deux études les plus rigoureuses sur le besoin de SPP et le fardeau des symptômes de la GCSH. En 2015, leurs premiers travaux consistaient en une étude de cohorte longitudinale visant à observer les répercussions la GCSH sur la qualité de vie des patients en hématologie (greffe autologue, $n=30$; allogreffe 
d'intensité réduite, $n=30$; allogreffe myéloablative, $n=30$ ) et de leurs proches aidants $(n=47)$. El-Jawahri et collaborateurs (2015) ont constaté que le pourcentage de patients présentant des symptômes dépressifs statistiquement significatifs doublait entre l'évaluation initiale et le huitième jour post-GCSH (passant de $15,6 \%$ à 37,8 \%; $n=72 ; p=.005$ ); le pourcentage de patients répondant aux critères diagnostiques de dépression majeure augmentait également (passant de 7,8 \% à 36,7 \%; $n=72 ; p=.0001$ ). De plus, ils ont noté une diminution (de 109,6 à 96,0; $p<.0001$ ) des scores de qualité de vie des patients, mesurés à l'aide de l'index d'évaluation fonctionnelle du traitement du cancer pour les greffes de moelle osseuse (Functional Assessment of Cancer Therapy-Bone Marrow Transplant Index) (El-Jawahri et al., 2015). Du côté des proches aidants, ils ont constaté un déclin statistiquement significatif de la qualité de vie $(p=.03)$, de la capacité physique fonctionnelle $(p=.02)$, de la santé générale $(p=.0007)$, de la vitalité $(p=.003)$, du fonctionnement social $(p=.004)$, du fonctionnement émotionnel $(p=.05)$, ainsi qu'une hausse des scores de dépression ( $p=.03$; El-Jawhari et al., 2015). El-Jawahri et collaborateurs (2015) ont aussi observé que les patients en hématologie recevant une GCSH (et leurs proches aidants) avaient besoin de soins de soutien pendant leur séjour à l'hôpital.

Dans un essai clinique randomisé plus récent, El-Jawahri et collaborateurs (2016) ont recueilli des données en faveur de l'intégration des SPP aux soins usuels des patients en hématologie recevant une GCSH; pour ce faire, ils ont réparti aléatoirement 160 patients en hématologie et 94 proches aidants entre un groupe recevant des SPP (patients $n=81$; proches aidants $n=49$ ) et un autre bénéficiant des soins usuels (patients $n=79$; proches aidants $n=45$ ). Deux semaines après la GCSH, la qualité de vie des patients ( $p=.02$; IC $95 \%, 1,27$ à 14,19), l'anxiété $(p<.001$; IC $95 \%,-3,22$ à -1,29), et le fardeau des symptômes $(p=.02$; IC $95 \%,-11,46$ à $-1,05)$ ont montré une amélioration statistiquement significative dans le groupe recevant des SPP (El-Jawahri et al., 2016). Après trois mois, les scores mesurant le trouble de stress post-traumatique chez les patients recevant des SPP présentaient aussi une amélioration statistiquement significative ( $p=.002$; IC $95 \%$-7.12 à -1.58; El-Jawahri et al., 2016). Pourtant, malgré les avantages mis en évidence dans la littérature, recourir systématiquement aux services de soins palliatifs ne constitue pas la norme de soins de la majorité des centres de greffe, particulièrement au Canada.

\section{OBSTACLES ET FACTEURS FACILITANTS L'INTÉGRATION DES SOINS PALLIATIFS PRÉCOCES}

Beaucoup de facteurs font obstacle à l'intégration des SPP pour les receveurs d'une GCSH, notamment les préjugés sur les soins palliatifs, l'évolution imprévisible des hémopathies malignes, les philosophies différentes de l'oncologie et des soins palliatifs, et l'incertitude quant au moment propice pour faire appel aux SPP (Kain et Eisenhauer, 2016; Pfeil, Laryionava, Reiter-Theil, Hiddmann et Winkler, 2015; Roeland et $\mathrm{Ku}, 2015)$. Bien souvent, les hématologues estiment que les soins palliatifs ne sont utiles qu'en fin de vie et peuvent par conséquent être moins disposés à y recourir (LeBlanc et al.,
2015). De plus, les services de soins palliatifs ne sont pas considérés comme ayant un rôle à jouer dans l'approche curative de la GCSH, ces services étant souvent associés à une «sentence de mort » (Chung et al., 2009, p. 265; Levine et al., 2017). L'hématologie et la GCSH ont habituellement une visée curative, approche qui ne semble pas cadrer avec les soins palliatifs. Dans l'avenir, le défi sera de transformer les perceptions et de rebâtir la structure pour soutenir les patients en hématologie et les receveurs d'une GCSH.

Dans l'espoir d'optimiser la prestation des SPP, la créativité constitue le mot d'ordre pour atténuer les obstacles aux soins palliatifs précoces et faciliter l'établissement d'un partenariat entre les équipes de GCSH et les soins palliatifs. Il faudrait, par exemple, identifier les professions de la santé contribuant au succès de la mise en œuvre des SPP (Dyar et al., 2012; Owens et al., 2012). Les IP sont des professionnelles autonomes et rentables qui possèdent d'excellentes aptitudes cliniques et collaboratives; elles sont donc bien placées pour combler le fossé et offrir des SPP aux receveurs d'une GCSH (Bakitas et al., 2015; Canadian Nurses Association/Association des infirmières et infirmiers du Canada, 2018; Deitrick et al., 2011; Fox, 2014; Kennedy, BrooksYoung, Nicol, Campbell et Brunton, 2015; Walling et al., 2017). L'intégration d'une IP spécialisée en soins palliatifs travaillant exclusivement avec l'équipe d'hématologie et de GCSH favoriserait le recours aux SPP pour les patients en hématologie et receveurs de GCSH.

\section{DES SOINS PALLIATIFS PAR DES INFIRMIĖRES PRATICIENNES}

Selon plusieurs auteurs, les IP répondent efficacement aux besoins en soins palliatifs des patients en oncologie (Bakitas et al., 2015; Clark et al., 2017; Dyar et al., 2012; Mitchell et al., 2016; Owens et al., 2012). Par exemple, Dyar et collaborateurs (2012) ont réparti aléatoirement des patients entre un groupe recevant des soins de soutien dispensé par une IP $(n=12)$ et un autre groupe uniquement les soins usuels $(n=14)$; ils ont constaté une amélioration statistiquement significative de la qualité de vie émotionnelle $(\mathrm{p}=.0106)$ et mentale $(p=.0219)$ chez les patients ayant bénéficié des interventions de l'IP. De plus, intégrer des infirmières praticiennes en soins palliatifs peut réduire le nombre de visites aux urgences, permettre de mieux planifier les soins avancés, et augmenter le nombre de patients adressés aux maisons de soins palliatifs et aux services de soutien psychosocial (Owens et al., 2012; Walling et al., 2017). Walling et collaborateurs (2017) ont mené une étude de cohorte prospective pour comparer l'effet des seuls soins usuels $(n=2370)$ à celui des soins palliatifs dispensés en ambulatoire par les IP $(n=224)$ à des patientes atteintes de cancer $\mathrm{du}$ sein. Selon cette même étude, les patients du groupe suivi par l'infirmière praticienne étaient plus susceptibles d'avoir préparé leurs documents de planification des soins avancés $(30 \%$ $[n=224]$ contre $17 \%$ [ $n=2370] ; \mathrm{p}<.01$ ) ou d'avoir été dirigés vers des services de soutien psychosocial (46\% $[n=224]$ contre $16 \%[n=2370] ; p<.01)$ ou une maison de soins palliatifs $(53 \%$ [ $n=224]$ contre $23 \%$ [ $n=2370] ; p=.02$ ) comparativement au groupe ayant reçu les soins habituels (Walling et al., 2017). 
De leur côté, Owens et collaborateurs (2012) ont réalisé un projet pilote pour étudier l'effet des soins palliatifs dispensés en ambulatoire par des IP aux patients atteints d'un cancer $(n=49)$; ils ont montré que la présence des IP en soins palliatifs réduisait le nombre de visites aux urgences, qui est alors passé de 0,07 par semaine en 2009 à 0,04 visite par semaine en 2010 ( $p=.001)$ (Owens et al., 2012).

\section{ÉLABORATION D'UN CHEMINEMENT CLINIQUE}

Bien que les SPP aient déjà fait leurs preuves auprès des patients en oncologie, le principal défi, à l'heure actuelle, est d'en optimiser la prestation. Selon les résultats de recherche, les IP sont bien placées pour répondre à l'ensemble des besoins entourant la qualité de vie qui accompagnent un diagnostic de cancer avancé ou qui découlent de complications associées à la GCSH. Malheureusement, la recherche montre qu'il est peu probable que les patients souffrant d'une hémopathie maligne reçoivent des soins palliatifs spécialisés pendant leur hospitalisation pour la GCSH, d'où la nécessité d'élaborer une démarche clinique pour mettre en lien ces patients avec les services de soins palliatifs (LeBlanc et al., 2015; Harden et Schembri et al., 2016; Howell et al., 2010).

\section{Un cheminement clinique pour l'intégration des infirmières praticiennes aux soins palliatifs précoces}

Le protocole clinique NEST, pour Nurse-Practitioner-

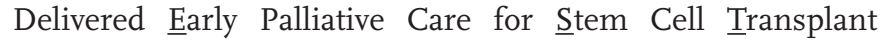
Patients (qui signifie « soins palliatifs précoces dispensés par les infirmières praticiennes aux patients recevant une greffe de cellules souches ») (voir figure 1) schématise l'intégration de l'IP spécialisée en soins palliatifs directement dans l'unité d'hématologie et de GCSH pour offrir des SPP aux patients dont l'hémopathie maligne est traitée par greffe. Selon ce cheminement clinique, tous les patients recevant une GCSH bénéficieront du soutien d'une IP en soins palliatifs. De plus, l'IP offrira également du soutien palliatif aux patients en hématologie qui présentent des symptômes encore présents ou non contrôlés, ou qui, avant même leur hospitalisation pour la GCSH, montrent des signes d'une piètre qualité de vie. Le recours potentiel aux SPP sera évalué à chaque admission, et plus souvent si nécessaire.

Même après recension des écrits, le cheminement clinique NEST semble être le premier protocole du genre élaboré expressément pour la prestation de SPP aux patients adultes hospitalisés en hématologie ou pour une GCSH. L'adoption de ce protocole pourrait influencer la culture traditionnellement axée sur la guérison de la GCSH et de l'hématologie, en plus de repousser les limites des soins prodigués aux patients greffés. Les centres qui mettent en œuvre ce cheminement clinique pourraient s'imposer comme chefs de file du domaine de l'hématologie et de la GCSH et favoriser l'adoption des SPP.

Facteurs facilitant l'intégration du cheminement clinique NEST

L'algorithme NEST permet de remédier à l'absence de SPP pour les receveurs d'une GCSH. Selon ce cheminement clinique, tous ces receveurs et leur famille sont vus par une IP spécialisée en soins palliatifs intégrés à l'équipe de greffe afin qu'ils soient bien outillés si le patient ne guérit pas, qu'il entre en fin de vie ou qu'il présente une morbidité associée à la maladie. L'IP prend en charge le fardeau des symptômes, l'atténuation de la souffrance et le maintien de la qualité de vie, ce qui permet aux équipes de GCSH de se concentrer sur les objectifs de traitements, la morbidité à long terme et la prévention de la mortalité. En restructurant ainsi la prestation des soins, on favorise l'établissement d'un partenariat entre les services de soins palliatifs et les équipes de GCSH pour offrir des soins complets aux patients (Levine et al., 2017).

Étant donné la nature imprévisible des hémopathies malignes, il n'est pas toujours évident de savoir quand le patient aura besoin de soins palliatifs et, parfois, il est trop tard pour en retirer des bienfaits intéressants. Certains signes indicateurs, définis dans les lignes directrices de l'ASCO, justifient le recours aux soins palliatifs, par exemple les hospitalisations fréquentes, la difficulté à contrôler les symptômes et la prise en charge complexe à cause, notamment, de l'administration d'antibiotiques, de l'aide à l'alimentation et du déclin du fonctionnement (Ferrell et al., 2017). La grande majorité des patients en GCSH et de nombreux patients en hématologie devraient être aiguillés vers les soins palliatifs en fonction de ces seuls indicateurs; malgré tout, le taux de renvoi demeure faible (Howell et al., 2010; LeBlanc et al., 2015). Lorsque le cheminement clinique NEST est intégré aux soins habituellement prodigués à ces patients, ces derniers peuvent alors profiter des bienfaits qu'apportent les SPP.

Le cheminement clinique NEST s'appuie sur l'expertise des IP, des professionnelles rentables et des spécialistes de la collaboration interdisciplinaire aux compétences riches et aux connaissances étendues. L'intégration d'une IP en soins palliatifs permettrait d'utiliser cette profession à sa pleine mesure et encouragerait davantage la création de postes d'IP dans le domaine des soins du cancer. En outre, le protocole NEST réduirait le stress et l'épuisement des professionnels de la santé en favorisant le partage des soins et en donnant aux membres de l'équipe la possibilité de prendre des décisions sur les cas complexes (D’Ambruso et al., 2016).

\section{Obstacles à l'intégration du cheminement clinique}

Comparativement aux modèles traditionnels, qui ne préconisent le recours aux services palliatifs qu'au besoin lorsque les patients présentent certains indicateurs (Levine et al., 2017), l'adoption du protocole NEST de soins palliatifs universels aurait comme inconvénient d'exiger nettement plus de ressources. De plus, selon la taille du centre de greffe et l'acuité des besoins des patients, il faudrait peut-être employer plusieurs IP. Toutefois, les IP sont des professionnelles rentables; cet obstacle pourrait donc être surmonté (O'Mahony et al., 2017). En soins palliatifs, les IP spécialisées ont déjà mené à des économies dans d'autres domaines, notamment dans les unités de soins intensifs. O’Mahony et collaborateurs (2017) ont estimé que l'intégration des IP en soins palliatifs, qui a entre autres permis de réduire la durée des hospitalisations et d'éliminer les examens inutiles, a apporté en six mois des économies atteignant 224500 \$ US.

Un autre facteur pourrait faire obstacle à l'adoption $\mathrm{du}$ cheminement clinique NEST : les équipes de GCSH, 
habituellement dirigées par un médecin, doivent être disposées à accueillir une IP en soins palliatifs. Mais cet obstacle serait surmonté si on met l'accent sur le partage de la responsabilité des soins. Les besoins des patients atteints de cancer sont complexes et il est irréaliste de s'attendre à ce que l'équipe d'hématologie ou de GCSH arrive à elle seule à combler tous leurs besoins physiques, psychosociaux, existentiels et spirituels (D’Ambruso et al., 2016). Si chaque sous-spécialité se concentrait sur son domaine d'expertise, on sauverait du temps et on améliorerait la qualité des soins.

\section{PISTES DE RECHERCHE}

D’autres études rigoureuses devront être menées pour analyser l'effet des services de soins palliatifs dispensés par les infirmières praticiennes aux patients recevant une GCSH. Différents projets seront nécessaires pour évaluer les modèles de soins qui intègrent directement les services de soins palliatifs aux unités hospitalières d'hématologie et de GCSH pour qu'ils fassent exclusivement partie des soins usuels prodigués. La recherche à venir pourrait également se pencher sur les

\section{RÉFÉRENCES}

Bakitas, M., Lyons, K. D., Hegel, M. T., Balan, S., Brokaw, F. C., Seville, J., Hull, J. G., Li, Z., Tosteson, T. D., Byock, I. R., \& Ahles, T. A. (2009). Effects of a palliative care intervention on clinical outcomes in patients with advanced cancer: The project ENABLE II randomized controlled trial. JAMA, 302(13), 741-749. https://doi. org/10.1001/jama.2009.1198

Bakitas, M. A., Tosteson, T. D., Li, Z., Lyons, K. D., Hull, J. G., Li, Z., Dionne-Odom, J. N., Frost, J., Dragnev, K. H., Hegel, M. T., Azuero, A., Ahles, T. A. (2015). Early versus delayed initiation of concurrent palliative oncology care: Patient outcomes in the ENABLE III randomized controlled trial. Journal of Clinical Oncology, 33(13), 1438-1445. https://doi.org/10.1200/jco.2014.58.6362

Canadian Nurses Association. (2018). Nurse practitioner. https://cnaaiic.ca/en/professional-development/advanced-nursing-practice/ nurse-practitioners

Canadian Medical Association. (2015). Palliative care: Canadian medical association's national call to action. https://www.cma.ca/Assets/assetslibrary/document/en/advocacy/palliative-care-report-online-e.pdf

Chung, H. M., Lyckholm, L. J., \& Smith, T. J. (2009). Palliative care in BMT. Bone Marrow Transplantation, 43(4), 265-273. https://doi. org/10.1038/bmt.2008.436

Clark, M. A., Ott, M., Rogers, M. L., Politi, M. C., Miller, S. C., Moynihan, L., Robison, K., Stuckey, A., \& Dizon, D. (2017). Advance care planning as a shared endeavor: Completion of ACP documents in a multidisciplinary cancer program. PsychoOncology, 26(1), 67-73. https://doi.org/10.1002/pon.4010

D’Ambruoso, S. F., Coscarelli, A., Hurvitz, S., Wenger, N., Coniglio, D., Donaldson, D., Pietras, C., \& Walling, A. M. (2016). Use of a shared mental model by a team composed of oncology, palliative care, and supportive care clinicians to facilitate shared decision making in a patient with advanced cancer. American Society of Clinical Oncology, 12(11), 1039-1045. https://doi.org/10.1200/ JOP.2016.013722

D’Souza, A., Lee, S., Zhu, X., \& Pasquini, M. (2017). Current use and trends in hematopoietic cell transplantation in the United States. Biology of Blood and Marrow Transplantation, 23(9), 1417-1421. https://doi.org/10.1016/j.bbmt.2017.05.035 avantages économiques des SPP dans les cas de GCSH, ainsi que sur les effets d'un partenariat entre les soins palliatifs et les équipes d'hématologie et de GCSH.

\section{CONCLUSION}

En conclusion, la GCSH, qui a pour but de guérir la maladie ou d'assurer la survie à long terme, est très intense pour les patients et leur famille. Ces traitements peuvent avoir des effets secondaires dévastateurs, entraîner de pénibles sentiments de peur et de stress, et même causer la mort. Et pourtant, bien que les soins palliatifs aient fait leurs preuves dans les soins du cancer, ils continuent d'être absents du traitement des patients recevant une GCSH. Le cheminement clinique NEST vise justement un partenariat entre les services de soins palliatifs et les équipes de GCSH. Il met l'accent sur le travail d'équipe et l'entraide interdisciplinaire pour offrir les meilleurs soins aux patients et aux familles. Son adoption est nécessaire si nous voulons accomplir de véritables progrès dans la prestation des SPP aux patients recevant une GCSH et les aider à maintenir une bonne qualité de vie, ce qui, au fond, est notre principal objectif.

Dans, M., Smith, T., Back, A., Baker, J. N., Bauman, J. R., Beck, A. C., Block, S., Campbell, T., Case, A. A., Dalal, S., Edwards, H., Fitch, T. R., Kapo, J., Kutner, J. S., Kvale, E., Miller, C., Misra, S., Mitchell, W., Portman, D. G., Spiegel, D., ... Scavone, J. L. (2017). NCCN guidelines insights: Palliative care, version 2.2017. The Official Journal of the National Comprehensive Cancer Network, 15(8), 989997. https://doi.org/10.6004/jnccn.2017.0132

Deitrick, L. M., Rockwell, E. H., Gratz, N., Davidson, C., Lukas, L., Stevens, D., Fitzgerald, G., Naugle, M., Wolf, J., \& Sikora, B. (2011). Delivering specialized palliative care in the community: A new role for nurse practitioners. Advances in Nursing Science, 34(4), 23-36. https://doi.org/10.1097/ANS.0b013e 318235834f

Dyar, S., Lesperance, M., Shannon, R., Sloan, J., \& Colon-Otero, G. (2012). A nurse practitioner directed intervention improves the quality of life of patients with metastatic cancer: Results of a randomized pilot study. Journal of Palliative Medicine, 15(8), 890895. https://doi.org/10. 1089/jpm.2012.0014

El-Jawahri, A. R., Traeger, L. N., Kuzmuk, K., Eusebio, J. R., Vandusen, H. B., Shin, J. A., Keenan, T., Gallagher, E. R., Greer, J. A., Pirl, W. F., Jackson, V. A., Ballen, K. K., Spitzer, T. R., Graubert, T. A., McAfee, S. L., Dey, B. R., Chen, Y. B., \& Temel, J. S. (2015). Quality of life and mood of patients and family caregivers during hospitalization for hematopoietic stem cell transplantation. Cancer, 121(6), 951-959. https://doi.org/10.1002/cncr.29149

El-Jawahri, A., LeBlanc, T., VanDusen, H., Traeger, L., Greer, J. A., Pirl, W. F., Jackson, V. A., Telles, J., Rhodes, A., Spitzer, T. R., McAfee, S., Chen, Y. A., Lee, S. S., \& Temel, J. S. (2016). Effect of inpatient palliative care on quality of life 2 weeks after hematopoietic stem cell transplantation: A randomized clinical trial. Journal of the American Medical Association, 316(20), 2094-2103. https://doi. org/10.1001/jama.2016.16786

Ferrell, B. R., Temel, J. S., Temin, S., Alesi, E. R., Balboni, T. A., Basch, E. M., Firn, J. I., Paice, J. A., Peppercorn, J. M., Phillips, T., Stovall, E. L., Zimmermann, C., \& Smith, T. J. (2017). Integration of palliative care into standard oncology care: American society of clinical oncology clinical practice guideline update. Journal of Clinical Oncology, 35(1), 96-112. https://doi.org/10.1200/ jco.2016.70.1474 
Fox, K. (2014). The role of the acute care nurse practitioner in the implementation of the commission on cancer's standards on palliative care. Clinical Journal of Oncology Nursing, 18(Suppl), 39-44. https://doi.org/10.1188/14.CJON.S1.39-44

Harden, K. L., \& Schembri, J. A. (2016). Early intervention with transplant recipients to improve access to and knowledge of palliative care. Clinical Journal of Oncology Nursing, 20(4), E88-92. https://doi.org/10.1188/16.CJON

Ho, H. T., Barbera, L., Saskin, R., Lu, H., Neville, B. A., \& Earle, C. C. (2011). Trends in the aggressiveness of end-of-life cancer care in the universal health care system of Ontario, Canada. Journal of Clinical Oncology, 29(12), 1587-1591.

Hochman, M. J., Yu, Y., Wolf, S. P., Samsa, G. P., Kamal, A. H., \& LeBlanc, T. W. (2018). Comparing the palliative care needs of patients with hematologic and solid malignancies. Journal of Pain and Symptom Management, 55(1), 82-88. https://doi.org/10.1016/j. jpainsymman .2017.08.030

Howell, D. A., Shellens, R., Roman, E., Garry, A. C., Patmore, R., \& Howard, M. R. (2010). Hematological malignancy: Are patients appropriately referred for specialist palliative and hospice care? A systematic review and meta-analysis of published literature. Palliative Medicine, 25(6), 630-640. https://doi. org/10.1177/0269216310391692

Hui, D., Kim, S. H., Roquemore, J., Dev, R., Chisholm, G., \& Bruera, E. (2014). Impact of timing and setting of palliative care referral on quality of end-of-life care in cancer patients. Cancer, 120(11), 1743-1749.

Kain, D. A., \& Eisenhauer, E. A. (2016). Early integration of palliative care into standard oncology care: Evidenced and overcoming barriers to implementation. Current Oncology, 23(6), 374-377. https://doi.org/10.3747/co.23.3404

Kennedy, C., Brooks Young, P., Nicol, J., Campbell, K., \& Gray Brunton, C. (2015). Fluid role boundaries: Exploring the contribution of the advanced nurse practitioner to multiprofessional palliative care. Journal of Clinical Nursing, 24(21/22), 3296-3305. https://doi.org/10.1111/jocn.12950

LeBlanc, T. W., O'Donnell, J. D., Crowley-Matoka, M., Rabow, M. W., Smith, C. B., White, D. B., Tiver, G. A., Arnold, R. M., \& Schenker, Y. (2015). Perceptions of palliative care among hematologic malignancy specialists: A mixed-methods study. Journal of Oncology Practice, 11(2), e230-238. https://doi.org/10.1200/ JOP.2014.001859

Levine, D. R., Baker, J. N., Wolfe, J., Lehmann, L. E., \& Ullrich, C. (2017). Strange bedfellows no more: How integrated stem-cell transplantation and palliative care programs can together improve end-of-life care. Journal of Clinical Oncology, 13(9), 569-577. https:// doi.org/10.1200/JOP.2017.021451

Loggers, E. T., LeBlanc, T. W., El-Jawahri, A., Fihn, J., Bumpus, M., David, J., Horak, P., \& Lee, S. J. (2016). Pre-transplantation supportive and palliative care consultation for high-risk hematopoietic cell transplantation patients. Biology of Blood and Marrow Transplantation, 22(7), 1299-1305. https://doi.org/10.1016/j. bbmt.2016.03.006

May, P., Garrido, M. M., Cassel, J. B., Kelley, A. S., Meier, D. E., Normand, C., Smith, T. J., \& Morrison, R. S. (2017). Cost analysis of a prospective multi-site cohort study of palliative care consultation teams for adults with advanced cancer: Where do cost-savings come from? Palliative Medicine, 31(4), 378-386. https:// doi.org/10.1177/0269216317690098
Mitchell, G. K., Senior, H. E., Bibo, M. P., Makoni, B., Young, S. N., Rosenberg, J. P., \& Yates, P. (2016). Evaluation of a pilot of nurse practitioner led, GP supported rural palliative care provision. BMC Palliative Care, 15, 1-11. https://doi.org/10.1186/s12904-016-0163-y

Owens, D., Eby, K., Burson, S., Green, M., McGoodwin, W., \& Isaac, M. (2012). Primary palliative care clinic pilot project demonstrates benefits of a nurse practitioner-directed clinic providing primary and palliative care. Journal of the American Academy of Nurse Practitioners, 24(1), 52-58. https://doi. org/10.1111/j.1745-7599.2011.00664.x

O’Mahony, S., Johnson, T. J., Amer, S., McHugh, M. E., McHenry, J., Fosler, L., \& Kvetan, V. (2017). Integration of palliative care advanced practice nurses into intensive care unit teams. American Journal of Hospice and Palliative Medicine, 34(4). 330-334. https:// doi.org/10.1177/1049909115627425

Pfeil, T. A., Laryionava, K., Reiter-Theil, S., Hiddemann, W., Winkler, E. C. (2015). What keeps oncologists from addressing palliative care early on with incurable cancer patients? An active stance seems key. The Oncologist, 20(1), 56-61. https://doi.org/10.1634/ theoncologist.2014-0031

Roeland, E., \& Ku, G. (2015). Spanning the canyon between stem cell transplantation and palliative care. American Society of Hematology, 15, 484-489. https://doi.org/10.1182/ asheducation-2015.1.484

Selvaggi, K. J., Vick, J. B., Jessell, S. A., Lister, J., Abrahm, J. L., \& Bernacki, R. (2014). Bridging the gap: A palliative care consultation service in a hematological malignancy-bone marrow transplant unit. The Journal of Community and Supportive Oncology, 12(2), 50-55. https://doi.org/10.12788/jcso.0015

Smith, T. J., Temin, S., Alesi, E. R., Abernethy, A. P., Balboni, T. A., Basch, E. M., Ferrell, B. R., Loscalzo, M., Meier, D. E., Paice, J. A., Peppercorn, J. M., Somerfield, M., Stovall, E., \& Von Roenn, J. H. (2012). American Society of Clinical Oncology provisional clinical opinion: the integration of palliative care into standard oncology care. Journal of Clinical Oncology, 30(8), 880-887. https://doi. org/10.1200/JCO.2011.38.5161

Temel, J. S., Greer, J. A., Muzikansky, A., Gallagher, E. R., Admane, S., Jackson, V. A., Dahlin, C. M., Blinderman, C. D., Jacobsen, J., Pirl, W. F., Billings, J. A., \& Lynch, T. J. (2010). Early palliative care for patients with metastatic non-small-cell lung cancer. The New England Journal of Medicine, 363, 733-742. https://doi.org/10.1056/ NEJMoa1000678

Temel, J. S., Greer, J. A., Admane, S., Gallagher, E. R., Jackson, V. A., Lynch, T. J., Lennes, I. T., Dahlin, C. M., \& Pirl, W. F. (2011). Longitudinal perceptions of prognosis and goals of therapy in patients with metastatic non-small-cell lung cancer: Results of a randomized study of early palliative care. Journal of Clinical Oncology, 29(17), 2319-2326. https://doi.org/10.1200/ JCO.2010.32.4459

Walling, A. M., D'Ambruoso, S. F., Malin, J. L., Hurvitz, S., Zisser, A., Coscarelli, A., Clarke, R., Hackbarth, A., Pietras, C., Watts, F., Ferrell, B., Skootsky, S., \& Wenger, N. S. (2017). Effect and efficiency of an embedded palliative care nurse practitioner in an oncology clinic. Journal of Oncology Practice, 13(9), 792-799. https:// doi.org/10.1200/JOP.2017.020990

Zimmermann, C., Swami, N., Krzyzanowska, M., Hannon, B., Leighl, N., Oza, A., Moore, M., Rydall, A., Rodin, G., Tannock, I., Donner, A., \& Lo, C. (2014). Early palliative care for patients with advanced cancer: A cluster-randomized controlled trial. The Lancet, 383(9930), 1721-1730. https://doi.org/10.1016/ S0140-6736(13)62416-2 